\title{
A HIGH POWER LNA LASER FOR APPLICATION TO A NEW POLARIZED ELECTRON SOURCE
}

\author{
S. ESSABAA, J. ARIANER, I. BRISSAUD, H. HUMBLOT, M. LEDUC* and \\ L.D. SCHEARER ** \\ Institut de Physique Nucléaire, F-91406 Orsay cedex, France
}

\begin{abstract}
The recent development of high energy electron accelerators has generated a renewed interest in high current, high polarization electron sources. We have investigated several modifications to a method based on a pumped helium afterglow from which we expect improvements over the performances. These ones include the development of a high power, tunable LNA laser and the application of a new optical pumping scheme to the metastable helium atoms.
\end{abstract}

\section{1 - Description of the polarized electron source}

The spin polarized electron source developed at Orsay (Fig. 1) uses the chemi-ionization of $\mathrm{CO}_{2}$ gas by polarized metastable helium atoms to produce free polarized electrons. The extraction of electrons from a flowing helium afterglow was first reported by Walters and his collaborators at Rice University (1). Briefly a microwave discharge is used to generate $\mathrm{He}\left(2^{3} \mathrm{~S}\right)$ atoms. They are optically pumped using circularly polarized $2{ }^{3} S-2{ }^{3} P_{1}$ radiation to preferentially populate either $m_{s}=+1$ or $m_{s}=-1$ magnetic substates via the $\Delta \mathrm{mJ}=+1$ or -1 selection rule for right $\left(\sigma^{+}\right)$and left $\left(\sigma^{-}\right)$hand circularly polarized light respectively. Ionization is brought about by introducing $\mathrm{CO}_{2}$ gas into the stream of flowing polarized metastable atoms. In the chemi-ionization process these helium atoms fall back to the ground state leaving behind an oriented electrons and ions : $\mathrm{He}\left(2^{3} \mathrm{~S}\right) \uparrow \uparrow+\mathrm{CO}_{2} \rightarrow \mathrm{He}\left(1 \mathrm{~S}_{0}\right) \uparrow \downarrow+\mathrm{CO}_{2}^{+} \uparrow+\mathrm{e}^{-} \uparrow$.

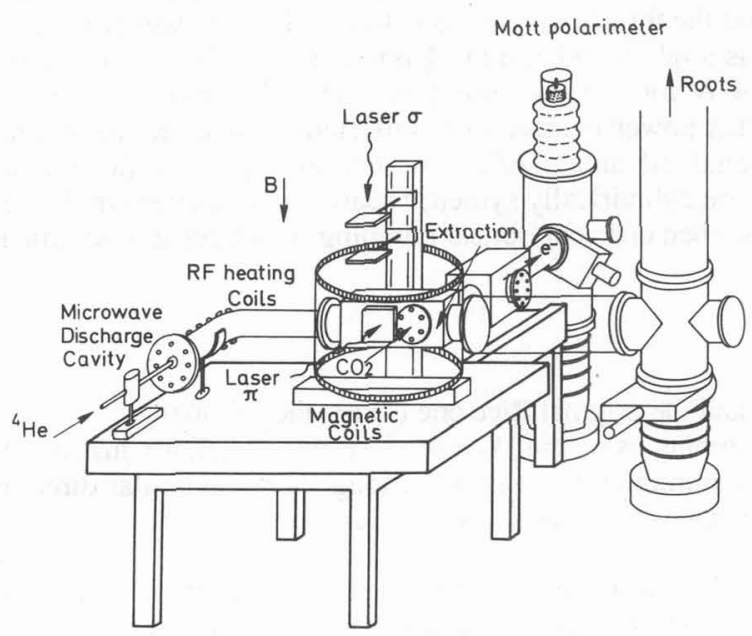

The polarized elections are then extracted and formed into a collimated beam by a suitable optics. The electron polarization is measured by a Mott polarimeter.

Eig. L: Schematic diagram of the Orsay polarized electron source 


\section{2 - The Orsay LNA laser}

To perform the optical pumping, we have developed an improved laser which has a large output efficiency and may be used to pump on the $2^{3} \mathrm{~S}-2^{3} \mathrm{P}$ transition $(\lambda=1.083 \mu \mathrm{m})$. The lasing material is an LNA crystal excited by krypton discharge lamps (2). The cavity is defined by two plane mirrors separated by 30 $\mathrm{cm}$. One of the mirrors is totally reflecting, the other one has a transmission of about $0.63 \%$ at $1.083 \mu \mathrm{m}$. Coarse tuning is accomplished with a $0.3 \mathrm{~mm}$ uncoated etalon. A second uncoated etalon of $1 \mathrm{~mm}$ thickness provides a narrower bandwidth. The linewidth is about $2.5 \mathrm{GHz}$, as mesured with a scanning Fabry-Perot spectrum analyzer. The mode structure consists of a large number of transverse modes within the measured linewidth. Such a line shape fills up the entire Doppler width of the atomic absorption line and provides efficient pumping even in low pressures where collisional redistribution of atomic velocities is weak.

To reduce the walk-off losses caused by the tilted etalons, we have applied a simple technique : we adjust the optical path length of the etalon by controlling its temperature (3). The etalon can be aligned in the cavity close to perpendicular incidence for maximum power, and the laser frequency can be tuned to the desired helium resonance, just by adjusting the temperature of the etalon with a Peltier element mounted on the etalon holders, without significant drop in laser power.

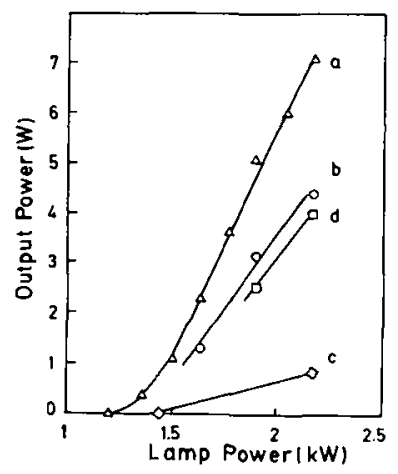

\section{Eig. 2 : Output power of the LNA laser versus electrical} lamp power.

a) No etalon; bandwidth is about $5 \mathrm{~nm}$.

b) With 2 etalons near normal incidence for optimum power; the laser linewidth is $2.5 \mathrm{GHz}$.

c) Laser tuned to $\mathrm{He}\left(2^{3} P_{0}\right)$ resonance by tilting both etalons.

d) Laser tuned to $\mathrm{He}\left(2^{3} P_{o}\right)$ resonance by thermal tuning of the I mm etalon.

When the laser frequency is tuned to the helium resonance line $(1.083 \mu \mathrm{m})$ by tilting both etalons, the power drops from $7 \mathrm{~W}$ to slightly below $1 \mathrm{~W}$ and the threshold rises to $1.44 \mathrm{KW}$ lamp power (fig. 2, curve c). Neverthless, when only the $0.3 \mathrm{~mm}$ etalon is angle tuned and the $1 \mathrm{~mm}$ etalon is thermally tuned, the output power reaches the maximum value of $4 \mathrm{~W}$ for $2.2 \mathrm{~kW}$ lamp power (fig. 2, curve d). The output gives over $5 \mathrm{~W}$ when both etalons are tuned. This power is more than sufficient to saturate the attainable polarization of the ${ }^{4} \mathrm{He}$ metastables. An additional advantage of the thermal tuning technique is that the quasi-normal position of the etalon leads to a more cylindrically symetric spatial mode pattern in the beam. This geometry is desirable in many experiments when efficient optical pumping in a large gas volume is to be performed.

\section{3 - The Orsay electron source}

The main differences between the Orsay source and the original Rice one (4) are the following :

i) At Orsay we apply a new scheme of optical pumping using the $D_{0}$ resonance line of helium instead of $D_{1}$ by combining $\pi$ and $\sigma$ polarized light; then the pumping beam is split along 2 perpendicular directions. This would result in a $100 \%$ polarization of $2^{3} \mathrm{~S}$ state, as it was shown by Schearer (5).

ii) Because of the large frequency width of the laser and of the high energy density, the irradiation region is shortened. The pumping time is much lower than the metastable relaxation time : these effects reduce the metastable depolarization by diffusion. 
iii) We have increased the flow velocity of the afterglow by using high speed mechanical pumps ; this yields high metastable densities by reducing the loss of metastabies diffusing to the walls.

\section{4 - Preliminary results}

Figure 3 shows the density of metastable atoms in the alfterglow. This density was measured by absorption of light tuned on $D_{2}$ resonance transition $\left(2^{3} S-2^{3} P_{2}\right)$; this light is delivered by another single mode LNA laser (6). This second laser has been built to probe the polarization of metastable atoms. For a $0.05-0.15$ mbar pressure range the mean density is about $10^{10} \mathrm{at.cm}^{-3}$. In a first set of measurements the extracted electron current reaches $35 \mu \mathrm{A}$ at $0.20 \mathrm{mbar}$ (fig. 3) and it can be increased by increasing the helium and $\mathrm{CO}_{2}$ flow pressure. Otherwise, this current must be higher by optimizing the extraction efficiency which is still very low $(<10 \%)$.

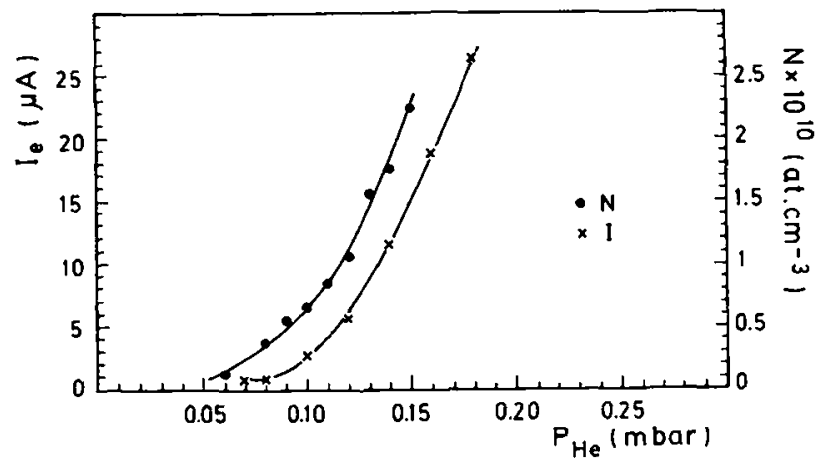

Eig 3 : Density of metastable
atoms and electron current as
a function of helium pressure.

In principle with this method the electron polarization can reach $100 \%$, as the helium atoms can be polarized to $100 \%$ and the chemi-ionization process was proved not to degrade this value (7). In our experiment, we do observe that their polarization increases when the $\pi$ pumping beam is added to the $\sigma$ one. We assume that the (40-50\%) electron polarization observed (fig. 4) is related to a degradation close to the extraction region (magnetic depolarization, collection of spurious electrons etc...). We are currently working to improve this part of the set-up. Indeed we believe that the metastable polarization is already close to $100 \%$. Furthermore we observe a saturation of the polarization signals with the laser intensity. Further improvements are expected from a better adjustment of the overlap between the two pump beams.

In conclusion higher beam currents and larger electron polarizations are expected from these improvements. $90 \%$ polarization and 50 to $100 \mu \mathrm{A}$ currents must be attained.

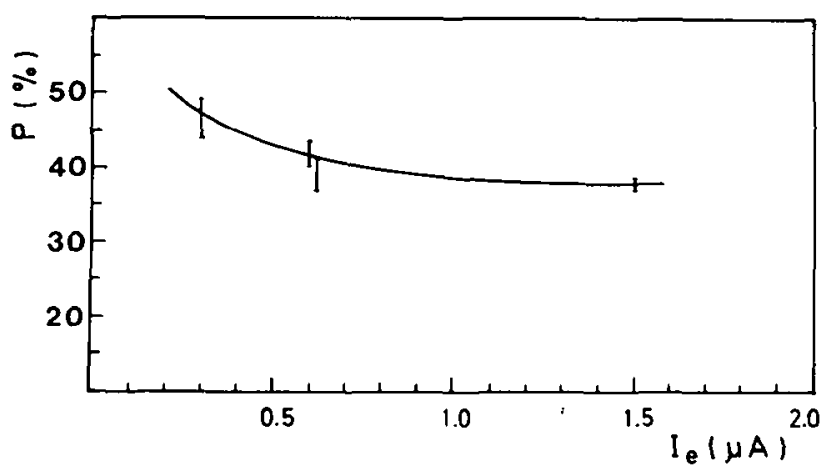

Eig. 4 : Electron polarization as a function of electron current (preliminary results). 
The authors wish to thank J. Obert , J.C. Putaux, and the separator group for their technical support. They would like to express their appreciation of discussions and encouragements given by G.K. Walters and the Rice University group, C.G. Aminoff and G. Lampel. The assistance of S. Pinard was very appreciated during the data acquisition.

* Laboratoire de Spectroscopie Hertzienne de lENS, 75014 Paris Cedex 05, France

** Permanent address : Physics Department, University of Missouri-Rolla, MO 65401, USA

References

/1/ RUTHERFORD G. H., RATLIFF J.M., TANG F.C., DUNNING F.B., and WALTERS G.K., Rev. Sci. Instr. 61 (1990) 1460 and references herein.

121 AMINOFF C.G., LARAT C., LEDUC M., and LALOE F., Rev. Phys.Appl. 24 (1989) 827.

B/ AMINOFF C.G., ESSABAA S., BRISSAUD I., and ARIANER J., submitted to publication.

14/ ESSABAA S., AMINOFF C.G., ARIANER J., and BRISSAUD I., Proc. the $9^{\text {th }}$ Intern.Symp. High. Ener. Phys., Bonn (1990), Springer-Verlag Edt., to be published.

/5/ SCHEARER L.D. and PADETHA TIN, Phys. Rev. A $\underline{42}$ (1990) 4028.

16 SCHEARER L.D., ESSABAA S., and LEDUC M., contribution to this conference.

n) KELIHER P.J., DUNNING F.B., O'NEILL M.R., RUDEL R.D., WALTERS G.K., Phys. Rev. A 11 (1975) 1271. 